\title{
Internal Branding and Leader-Member Exchange: Role of Cultural Capital in Employee's Service Delivery Behaviour in Healthcare Sector: An Abstract
}

\author{
Puja Dhawan and Daniel Prior
}

\begin{abstract}
The idea of a strong bond between culture and health (see Bourdieu 1985) is explored in detail by investigating the role of cultural capital in the internal brand-building practices of leaders and members working in the healthcare sector. However, service employees often fluctuate in their delivery of the brand promise, leaving firms with less direct control over service quality. Besides, internal branding is a predictor of service quality. Nevertheless, the issue of how to get service employees to act in a way that enhances service quality, considering they may not share same cultural capital, is clearly of pivotal importance. Previous studies have identified the role of leader in implementing internal branding efforts; however, limited research explains how leader-member exchange influences internal branding outcomes, in view of cultural capital, especially in the healthcare sector. It is, therefore, the focus of the present study. Drawing on interviews with 36 respondents from India, the study addresses this issue. Firstly, this study identifies that cultural capital influences leader-member exchange. Cultural capital similarities in age, experience and education among leaders and members encourage transformational leadership styles. On the other hand, cultural capital dissimilarities in age, experience and education encourage transactional leadership. Secondly, the study highlights that leader-member cultural capital similarity is more likely to facilitate internal branding through communication and training and hence enhance service quality. Thirdly, employees' service delivery behaviour in public healthcare is more likely to be affected by low resource availability and minimal incentives schemes wherein leaders lack financial incentive offers. The study, therefore, offers knowledge about managing leader-member exchange and thereby facilitating internal branding outcomes.
\end{abstract}

\footnotetext{
P. Dhawan $\bullet$ D. Prior $(\bowtie)$

University of New South Wales, Canberra, Australia

e-mail: pujanet@gmail.com; d.prior@adfa.edu.au
} 